\title{
Bidirectional OFDM-WDM-PON System Employing 16-QAM Intensity Modulated OFDM Downstream and OOK Modulated Upstream
}

\author{
Fady I. El-Nahal* \\ ${ }^{1}$ Institute for Communications Engineering, Technical University of Munich, 80290 Munich, Germany \\ Received April 28, 2008; accepted June 29, 2016; published June 30, 2016
}

\begin{abstract}
We have investigated an orthogonal frequency-division multiplexing (OFDM) wavelength-division-multiplexing (WDM) passive optical network (PON) with centralized lightwave and direct detection. The system was demonstrated for $10 \mathrm{Gbps} 16$ quadrature amplitude modulation (16-QAM) intensity-modulated OFDM downstream signals and $2.5 \mathrm{Gbps}$ On-off keying (OOK) upstream signals respectively. A wavelength-reuse scheme is employed to carry the upstream data by using a mach-zehnder modulator (MZM) as an intensity modulator at the optical network unit (ONU). The proposed scheme offers a promising solution for future PONs.
\end{abstract}

The rapid growth of personal bandwidth demand is continuing to fuel the penetration of fiber into the access network section. The time-division-multiplexed passive optical network (TDM-PON) has already been deployed to meet the huge bandwidth demand of broadband services. Although TDM-PONs are cost effective, they suffer from the bandwidth sharing nature. The wavelength division multiplexed passive optical network (WDMPON) has been proposed to increase the bandwidth utilization of optical fiber. WDM-PON is emerging as a promising key technology for the future fiber-to- thehome (FTTH) [1-4].

Several modulation schemes have been demonstrated such as downstream and upstream on-off keying (OOK), downstream differential phase-shift keying (DPSK) and upstream on-off keying (OOK), downstream DPSK and upstream DPSK, downstream QPSK and upstream OOK [5-9]. WDM PON can also be realized by using the optical orthogonal-frequency-division- multiplexing (OFDM) technique [10-14]. There has been an increased interest in Orthogonal Frequency Division Multiplexing (OFDM) in optical communication systems due to the need for higher data rates. OFDM is an efficient modulation technology which offers high spectral efficiency and ability to overcome limitations in optical fiber communication systems, such as relative intensity noise, modal dispersion, chromatic dispersion (CD), and polarization mode dispersion (PMD) [15-16]. Therefore OFDM modulation could be used in WDM-PON to increase bandwidth utilization and extend transmission distance.

Although coherent detection offers higher receiver sensitivity compared with the direct detection (DD) receiver. Direct-detection optical OFDM can considerably simplify the system complexity and reduce the phase noise and frequency offset [17].A low cost optical network unit (ONU) for WDM-PON is desirable. The wavelength reused technique is a cost-effective solution in WDMPON systems. There is no optical source in ONU, downstream wavelength signals are re-modulated with uplink data and then sent upstream towards the central office (CO). In these systems, both upstream and downstream channels use the same wavelength for improving wavelength utilization efficiency [18-21]. The Mach-Zehnder Modulator (MZM) can be used to remodulate the downstream signal at ONU and send it back to $\mathrm{CO}$ as an upstream signal [21].

In this article, 70-km-reach colorless OFDM-WDMPON with centralized lightwave and direct detection has been evaluated. The downstream signal with $10-\mathrm{Gb} / \mathrm{s}$ intensity modulated quadrature amplitude modulation (16QAM) OFDM carried by a 12-GHz sinusoidal wave and upstream with a $2.5-\mathrm{Gb} / \mathrm{s}$ OOK signal has been demonstrated.

Figure 1 shows the proposed WDM-OFDM-PON architecture using downstream OFDM and upstream OOK signals. At the central office, there are $\mathrm{N}$ distributed laser sources, followed by intensity modulators (IMs). The IM is used to create up-converted OFDM intensity modulated signals for WDM-OFDM downstream transmission. 128 subcarriers of 16QAM format are used with the Fast Fourier transform (FFT) size of 512 and Cyclic prefix (CP) of 10. On each WDM channel (wavelength), a 10Gbit/s OFDM-16QAM signal is up-converted to high radio frequency $(\mathrm{RF})$ carrier frequency $(12 \mathrm{GHz})$, by an analog mixer with an RF source.

${ }^{*}$ E-mail: fady.el-nahal@tum.de 


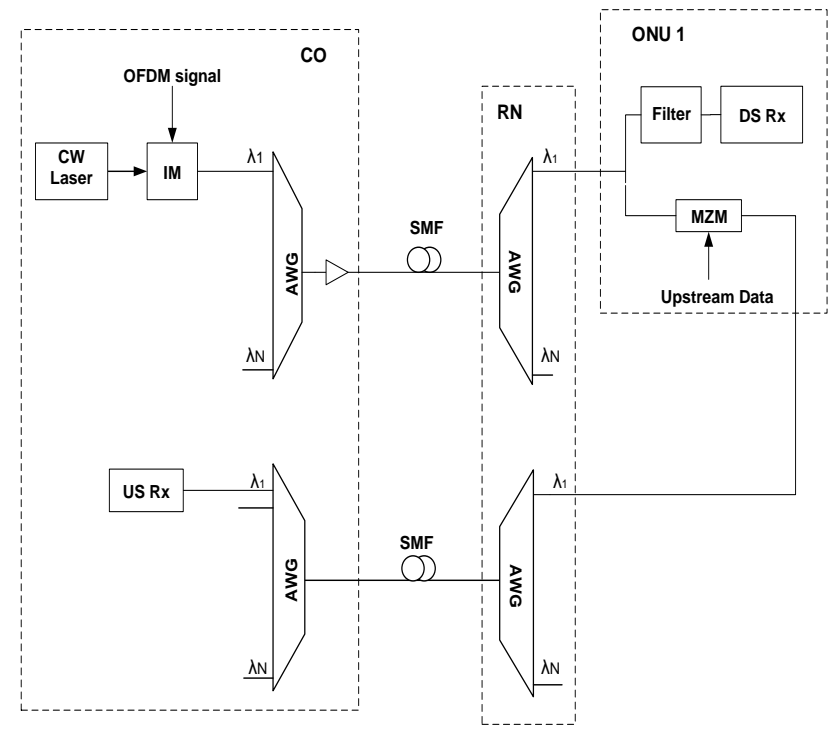

Fig. 1: The proposed OFDM-WDM-PON architecture.

The WDM channels are multiplexed using an arrayed waveguide grating $(\mathrm{AWG})$ at $\mathrm{CO}$ and the aggregate signal is amplified by EDFA (NF=4) and sent over $70 \mathrm{~km}$ singlemode fiber (SMF) for downstream transmission. In the remote node (RN), OFDM-WDM signals are demultiplexed by AWG where various wavelength signals are sent to different ONUs. At ONU, using a 3-dB optical splitter, half of the OFDM modulated signal is passed to a narrowband optical filter (OF) to remove excess ASE noise, and then down-converted before direct detection by a downstream receiver. No optical pre-amplifier was used in detection. For up-link, the other half of the downstream OFDM modulated signal is directly re-modulated by an IM (MZM) to generate the $2.5 \mathrm{~Gb} / \mathrm{s} \mathrm{NRZ} \mathrm{upstream} \mathrm{ON-}$ OFF Keying (OOK) signal. The re-modulated OOK signal is then launched back through $\mathrm{AWG}$ at $\mathrm{RN}$ via $70-\mathrm{km}$ SMF to $\mathrm{CO}$ where it is demultiplexed by $\mathrm{AWG}$ and received by a $2.5-\mathrm{GHz}$ receiver in $\mathrm{CO}$. Hence a centralized lightwave is achieved without the need for any additional light source in ONU.

The OFDM-WDM-PON system was simulated using a commercial package [22]. Figure 2 shows the bit-errorrate (BER) measurements and the corresponding constellations of the $10-\mathrm{Gb} / \mathrm{s}$ downstream for both 16 QAM and 4-QAM intensity modulated OFDM signals; while Fig. 3 shows the BER measurements of the corresponding $2.5-\mathrm{Gb} / \mathrm{s}$ upstream OOK signals. The OOK signal was generated from the re-modulation of the downstream OFDM signal.

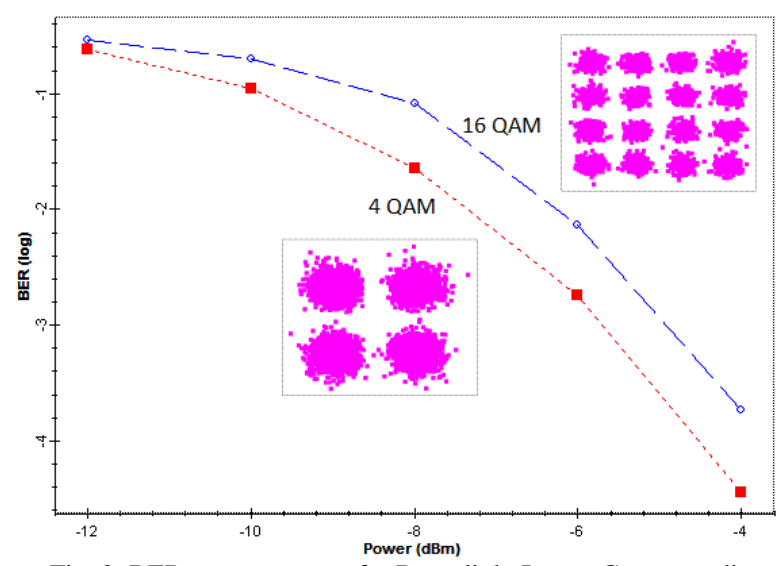

Fig. 2: BER measurements for Downlink. Insets: Corresponding constellation diagrams.

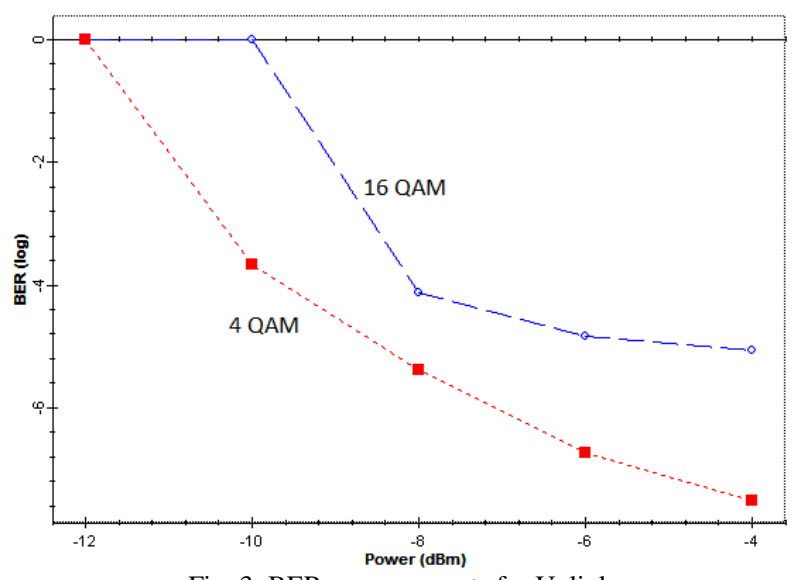

Fig. 3: BER measurements for Uplink.

It can be clearly seen that both uplink and downlink do provide good BER performance. However the 4-QAM OFDM outperforms the 16-QAM OFDM, which is expected as higher order QAMs are more susceptible to noise and transmission errors.

The bit-error-rate (BER) curves at back-to-back (B-TB) and after 70-km SMF and the corresponding constellations of downstream 16-QAM intensity modulated OFDM are shown in Fig. 4. The downlink 10 Gbps 16-QAM signals can achieve a BER performance of $9.3 \mathrm{e}^{-5}$ and the $70 \mathrm{~km}$ transmission curve is very close to that of the back to back. Therefore, a $70 \mathrm{~km} \mathrm{SMF}$ transmission can be achieved with negligible power penalty and without any dispersion compensation. Fig. 5 shows the BER measurements of the $2.5-\mathrm{Gb} / \mathrm{s}$ upstream OOK signal. It can be seen from the results that the uplink 2.5 Gbps OOK signals can achieve a $-19.2 \mathrm{dBm}$ receiver sensitivity at BER of $1 \mathrm{e}^{-5}$ and the power penalty increase with increasing received power, where it is about $2 \mathrm{~dB}$ after $70 \mathrm{~km}$ transmission due to dispersion and other nonlinear effects. 


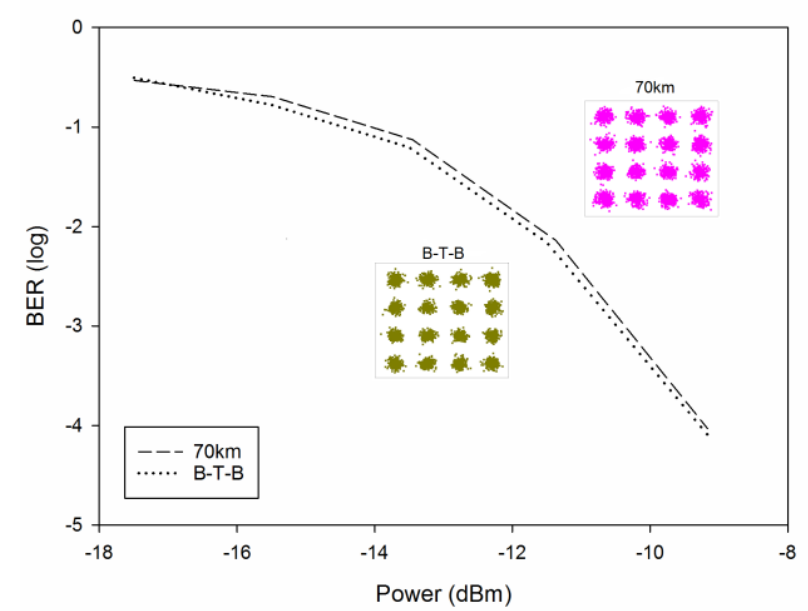

Fig. 4: BER measurements at back-to-back (B-T-B) and after 70-km for Downlink. Insets: Corresponding constellation diagrams.

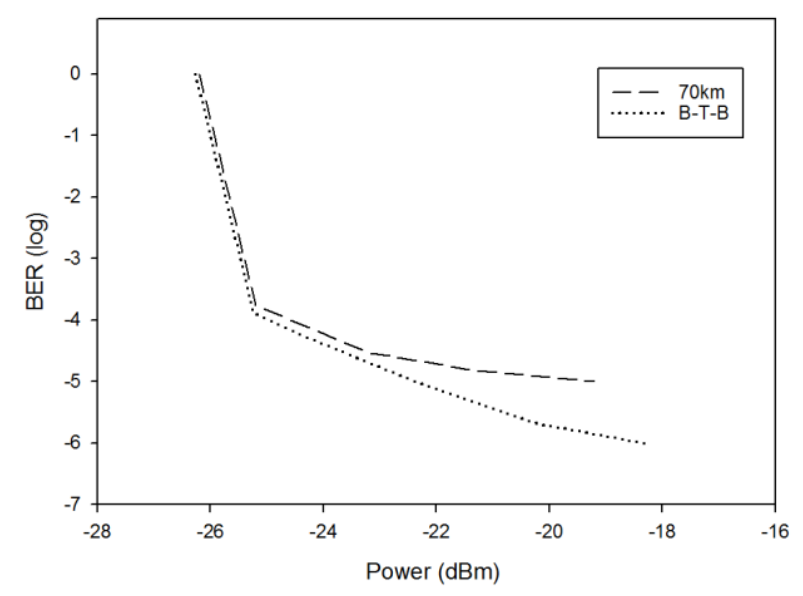

Fig. 5: BER measurements at back-to-back (B-T-B) and after 70-km for Uplink.

In conclusion, we have proposed and investigated an OFDM-WDM-PON system with centralized lightwave and direct detection. A 10Gbit/s 16-QAM intensity modulated OFDM has been transmitted over 70-km SMF as downstream. For upstream link, downstream OFDM signals are remodulated by MZM at $2.5 \mathrm{~Gb} / \mathrm{s}$. The results show that $10 \mathrm{Gbit} / \mathrm{s}$ downlink transmission with $9.3 \mathrm{e}^{-5}$ BER and $2.5 \mathrm{Gbit} / \mathrm{s}$ data rate for uplink transmission can be realized. The results show that the effect of dispersion is negligible, hence this scheme is a promising solution for next generation wavelength reuse WDM-PONs.

The author would like to acknowledge the Alexander von Humboldt Foundation, for their support. Also would like to acknowledge the help provided by Prof. Norbert Hanik.

\section{References}

[1] Elaine Wong, J. Lightwave Technol. 30(4), 597 (2012).

[2] F.T. An, D. Gutierrez, K.S. Kim, J.W. Lee, L.G. Kazovsky, IEEE Commun. Mag. 43(11), 540 (2005).

[3] S.-J. Park, C.-H. Lee, K.-T. Jeong, H.-J. Park, J.-G. Ahn, K.-H. Song, J. Lightwave Technol. 22, 2582 (2004).

[4] C.W. Chow, C.H. Yeh, IEEE Photon. J. 5(2), 7900407 (2013).

[5] J. Yu, M.-F. Huang, D. Qian, L. Chen, G.-K. Chang, IEEE Photon. Technol. Lett. 20(18), 1545 (2008).

[6] C.W. Chow, IEEE Photon. Technol. Lett. 20(1), 12 (2008).

[7] W. Hung, C.-K. Chan, L.-K. Chen, F. Tong, IEEE Phot. Techn. Lett. 15(10), 1630 (2003).

[8] N. Deng, C.K. Chan, L.K. Chen, Opt. Fiber Technol. 13, 18 (2007).

[9] G. Talli, C.W. Chow, E.K. MacHale, P.D. Townsend, J. Opt. Networking 6, 765 (2007).

[10] N. Cvijetic, J. Lightwave Techn. 30(4), 384 (2012).

[11] D. Qian, N. Cvijetic, J. Hu, T. Wang, Tech. Dig. OFC 2009, paper PDPD5 (2009).

[12] D. Qian, N. Cvijetic, Y.-K. Huang, J. Hu, T. Wang, Tech. Dig. ECOC 2009, paper PD3.3 (2009).

[13] M. Huang, D. Qian, N. Cvijetie, Proc. ECOC, Tu.5.C.1, Geneva (2011).

[14] T. Dong, Y. Bao, Y. Ji, A. Lau, Z. Li, C. Lu, IEEE Photonics Technol. Lett. 24(22), 2024 (2012).

[15] A. Chahine, U. Okonkwo, R. Ngah, IEEE International RF and Microwave Proceedings, 335-338 (2008).

[16] D. Qian, J. Yu, J. Hu, L. Zong, L. Xu, T. Wang, in OFC, San Diego, CA, Paper OMM3 (2008).

[17] B.J. Schmidt, A.J. Lowery, J. Armstrong, in OFC, Anaheim, CA, Paper PDP18 (2007).

[18] Y.C. Chung, Tech. Dig. ECOC 2011, Geneva, Paper Th.11.C.4 (2011)

[19] J.M. Kang, S.K. Han, IEEE Photon. Technol. Lett. 18(3), 502 (2006).

[20] C.H. Yeh, C.W. Chow, H.Y. Chen, J.Y. Sung, Y.L. Liu, Electron. Lett. 48(15), 940 (2012).

[21] C. Lei, M. Chen, H. Chen, S. Yang, S. Xie, Lasers and Electro-Optics (CLEO) 2015, pp. 1 - 2, (2015).

[22] OptiSystem Package from Optiwave. 